\title{
RENDIMENTO DE GRÃOS, COMPONENTES DO RENDIMENTO E ÍNDICE SPAD DO FEIJOEIRO (Phaseolus vulgaris L.) EM FUNÇÃO DE ÉPOCA DE APLICAÇÃO E DO PARCELAMENTO DA APLICAÇÃO FOLIAR DE MOLIBDÊNIO ${ }^{1}$
}

\author{
Grain yield, yield components and bean SPAD index of common bean \\ (Phaseolus vulgaris L.) in relation to time and partitioning of molybdenum foliar spray
}

André Assis Pires², Geraldo Antônio de Andrade Araújo ${ }^{3}$, Glauco Vieira Miranda ${ }^{3}$, Paulo Geraldo Berger ${ }^{3}$,
Alexandre Cunha de Barcellos Ferreira ${ }^{4}$, Poliana Daré Zampirolli ${ }^{5}$, Uberlando Tiburtino Leite RESUMO

Objetivando avaliar a influência da época de aplicação e do parcelamento da dose de molibdênio, aplicado via foliar sobre os componentes da produção e índice SPAD do feijoeiro (Phaseolus vulgaris L.), variedade Meia Noite, conduziram-se dois experimentos em área da Universidade Federal de Viçosa, no município de Coimbra, MG, semeados em março e julho de 2002. O delineamento experimental foi o de blocos casualizados, com dez tratamentos e quatro repetições. Os tratamentos avaliados foram: 1- testemunha, sem Mo; 2- $80 \mathrm{~g} \mathrm{ha}^{-1}$ de Mo aplicados 15 dias após a emergência (DAE); 3- $40 \mathrm{~g} \mathrm{ha}^{-1}$ de Mo aplicados 15 DAE e $40 \mathrm{~g} \mathrm{ha}^{-1} 20$ DAE; 4- $40 \mathrm{~g} \mathrm{ha}^{-1}$ de Mo 15 DAE e $40 \mathrm{~g} \mathrm{ha}^{-1} 25$ DAE; $5-40 \mathrm{~g} \mathrm{ha}^{-1}$ de Mo 15 DAE e $40 \mathrm{~g} \mathrm{ha}^{-1} 30$ DAE; 6- $80 \mathrm{~g} \mathrm{ha}^{-1}$ de Mo 20 DAE; 7- $40 \mathrm{~g} \mathrm{ha}^{-1}$ de Mo 20 DAE e $40 \mathrm{~g} \mathrm{ha}^{-1} 25$ DAE; 8- $40 \mathrm{~g} \mathrm{ha}^{-1}$ de Mo 20 DAE e $40 \mathrm{~g} \mathrm{ha}^{-1} 30$ DAE; 9- $80 \mathrm{~g} \mathrm{ha}^{-1}$ de Mo 25 DAE; 10- $40 \mathrm{~g} \mathrm{ha}^{-1}$ de Mo 25 DAE e $40 \mathrm{~g} \mathrm{ha}^{-1} 30$ DAE. Os dados dos dois experimentos foram submetidos a uma análise conjunta. A adubação foliar molíbdica aumenta a produtividade e o índice SPAD no cultivo de verãooutono com início aos 15 e 20 DAE, mas não aos 25 DAE. O parcelamento da dose não aumenta a produtividade. No cultivo de inverno-primavera, os tratamentos com molibdênio aumentam a produtividade.

Termos para indexação: Aplicação foliar, parcelamento, molibdênio, feijoeiro, Phaseolus vulgaris.

\begin{abstract}
A field trial was undertaken to evaluate the effect of the foliar application and dose partitioning of molybdenum on yield components and SPAD index of common bean (Phaseolus vulgaris L.), var. Meia Noite, sowed in March and in July of 2002, in an area of the Universidade Federal de Viçosa, Coimbra, MG. The randomized block experimental design was used with ten treatments and four replicates: The appraised treatments were: 1 - control, without Mo; 2 - $80 \mathrm{~g} \mathrm{ha}^{-1}$ Mo applied at 15 days after emergency (DAE); $3-40 \mathrm{~g} \mathrm{ha}^{-1}$ Mo applied at $15 \mathrm{DAE}$, and 40g at $20 \mathrm{DAE} ; 4-40 \mathrm{~g} \mathrm{ha}^{-1}$ Mo applied at 15 DAE, and $40 \mathrm{~g}$ $\mathrm{ha}^{-1}$ Mo at 25 DAE; 5 - $40 \mathrm{~g} \mathrm{ha}^{-1}$ Mo applied at 15 DAE, and $40 \mathrm{~g} \mathrm{ha}^{-1}$ Mo at $30 \mathrm{DAE} ; 6$ - $80 \mathrm{~g} \mathrm{ha}^{-1}$ Mo applied at 20 DAE; 7 - 40 $\mathrm{g} \mathrm{ha}^{-1}$ Mo applied at $20 \mathrm{DAE}$, and $40 \mathrm{~g} \mathrm{ha}^{-1}$ Mo at $25 \mathrm{DAE} ; 8-40 \mathrm{~g} \mathrm{ha}^{-1}$ Mo applied at 20 DAE, and $40 \mathrm{~g} \mathrm{ha}^{-1}$ Mo at $30 \mathrm{DAE} ; 9$ $80 \mathrm{~g} \mathrm{ha}^{-1}$ Mo applied at $25 \mathrm{DAE} ; 10-40 \mathrm{~g} \mathrm{ha}^{-1}$ Mo applied at $25 \mathrm{DAE}$, and $40 \mathrm{~g} \mathrm{ha}^{-1} \mathrm{Mo}$ at $30 \mathrm{DAE}$. ). Data of two trials were submitted to combined analysis. Molybdenum foliar spray increased yield and SPAD index in summer-autumn cultivation when started 15 or 20 DAE, but not 25 DAE. Dose partitioning had no effect on yield increase yield. In winter-spring cultivation, all molybdenum treatments increased yield.
\end{abstract}

Index terms: Application foliar, portioning, molybdenum, common bean, Phaseolus vulgaris.

(Recebido para publicação em 11 de setembro de 2003 e aprovado em 16 de junho de 2004)

\section{INTRODUÇÃO}

$\mathrm{O}$ nitrogênio $(\mathrm{N})$ é um dos nutrientes mais importantes na nutrição do feijoeiro, pois é constituinte básico da clorofila, dos aminoácidos, das proteínas, dos ácidos nucléicos e de outros compostos no metabolismo da planta, de modo que a produtividade dessa cultura está diretamente relacionada à sua nutrição nitrogenada (VIEIRA et al., 1992; AMANE et al., 1999). O metabolismo do $\mathrm{N}$ pode ser seriamente prejudicado pela deficiência de molibdênio (Mo) na planta, pois esse micronutriente faz parte das enzimas nitrogenase, essencial

\footnotetext{
1. Parte da tese apresentada à UFV - 36571-000 - Viçosa, MG, pelo primeiro autor, para obtenção do grau de Mestre em Fitotecnia na área de Nutrição Mineral de Plantas.

2. Engenheiro Agrônomo, Ms., Departamento de Fitotecnia, UFV, assires@bol.com.br.

3. Professores do Departamento de Fitotecnia, UFV.

4. Engenheiro Agrônomo, Ds., Pesquisador EPAMIG/CTZM, 36571-000 - Viçosa, MG.

5. Engenheira Agrônoma, mestranda no Centro de Ciências e Tecnologias Agropecuárias, Universidade Estadual do Norte-Fluminense, Campos dos Goytacazes, RJ.

6. Engenheiro Agrônomo, Doutorando do Departamento de Fitotecnia, UFV.
} 
para a fixação biológica do $\mathrm{N}_{2}$ pelo Rhizobium, e redutase do nitrato, indispensável para o aproveitamento do $\mathrm{N}$-nítrico absorvido pela planta. Assim, a carência de Mo provoca no feijoeiro sintomas semelhantes àqueles da deficiência de $\mathrm{N}$, ou seja, plantas com crescimento reduzido e folhas cloróticas.

$\mathrm{Na}$ maioria dos ensaios de adubação foliar com Mo, realizados na Zona da Mata de Minas Gerais, tem sido observado aumento de produção do feijoeiro (VIEIRA et al., 1992; BERGER et al., 1996; COELHO et al., 1998; AMANE et al., 1999; ARAÚJO, 2000; PESSOA et al., 2000), sendo as doses de 80 a $90 \mathrm{~g} \mathrm{ha}^{-1}$, aplicadas entre 14 e 28 dias após a emergência das plântulas, as que permitem as mais altas produções (BERGER et al., 1996). Aumentos substanciais de produtividade poderão, ainda, ser obtidos com o parcelamento da dose recomendada, já que esse procedimento promove maior eficiência de absorção e de uso do nutriente pela planta, contribuindo, desse modo, para ganhos em rendimento. Contudo, não há informações concretas sobre esse manejo da adubação molíbdica no feijoeiro.

Dessa forma, foram conduzidos dois experimentos em diferentes épocas de plantio, objetivando determinar a influência da época de aplicação e do parcelamento da dose de Mo aplicado via foliar sobre os componentes de produção e sobre o índice SPAD do feijoeiro (Phaseolus vulgaris L.).

\section{MATERIAL E MÉTODOS}

Foram realizados dois experimentos - um no período de verão-outono, com semeadura em março de 2002 (experimento I), e o outro no período de invernoprimavera, com semeadura em julho de 2002 (experimento II), utilizando-se em ambos a variedade Meia Noite. Os experimentos foram conduzidos em condições de campo, na área experimental do Departamento de Fitotecnia da Universidade Federal de Viçosa, em Coimbra, MG. O solo é classificado como Argissolo Vermelho-Amarelo Distrófico, que foi previamente amostrado à profundidade de $0-20 \mathrm{~cm}$, obtendo-se no experimento I: $\mathrm{pH}$ em água $(1: 2,5)=5,8 ; \mathrm{Al}^{3+}=0 \mathrm{cmol}_{\mathrm{c}} \mathrm{dm}^{-3}$; $\mathrm{Ca}^{2+}=1,54 \mathrm{cmol}_{\mathrm{c}} \mathrm{dm}^{-3} ; \mathrm{Mg}^{2+}=0,56 \mathrm{cmol}_{\mathrm{c}} \mathrm{dm}^{-3} ; \mathrm{K}=60$ $\mathrm{mg} \mathrm{dm}^{-3} ; \mathrm{P}=6 \mathrm{mg} \mathrm{dm}^{-3}$; Mo $=0,80 \mathrm{mg} \mathrm{dm}^{-3}$ (extrator Mehlich-3) ; $\mathrm{C}=1,46 \mathrm{dag} \mathrm{kg}^{-1}$; CTC efetiva $=2,25$ $\mathrm{cmol}_{\mathrm{c}} \mathrm{dm}^{-3} ;$ CTC total $=3,73 \mathrm{cmol}_{\mathrm{c}} \mathrm{dm}^{-3} ; \mathrm{V}=60 \%$; e classificação textural $=$ argiloso, e no experimento II: pH em água $(1: 2,5)=5,3 ; \mathrm{Al}^{3+}=0 \mathrm{cmol}_{\mathrm{c}} \mathrm{dm}^{-3} ; \mathrm{Ca}^{2+}=$ $1,23 \mathrm{cmol}_{\mathrm{c}} \mathrm{dm}^{-3} ; \mathrm{Mg}^{2+}=0,45 \mathrm{cmol}_{\mathrm{c}} \mathrm{dm}^{-3} ; \mathrm{K}=60 \mathrm{mg}$ $\mathrm{dm}^{-3} ; \mathrm{P}=6 \mathrm{mg} \mathrm{dm}^{-3} ; \quad \mathrm{Mo}=0,85 \mathrm{mg} \mathrm{dm}^{-3}$ (extrator
Mehlich-3); $\mathrm{C}=1,74 \mathrm{dag} \mathrm{\textrm {kg } ^ { - 1 }}$; CTC efetiva $=1,90$ $\mathrm{cmol}_{\mathrm{c}} \mathrm{dm}^{-3} ;$ CTC total $=3,93 \mathrm{cmol}_{\mathrm{c}} \mathrm{dm}^{-3} ; \mathrm{V}=46 \%$; e classificação textural $=$ argiloso.

Os experimentos foram organizados segundo delineamento experimental em blocos casualizados, com dez tratamentos e quatro repetições. Os tratamentos avaliados foram: 1- testemunha, sem Mo; 2- $80 \mathrm{~g} \mathrm{ha}^{-1} \mathrm{de}$ Mo aplicados aos 15 dias após a emergência (DAE); 3$40 \mathrm{~g} \mathrm{ha}^{-1}$ de Mo aplicados aos 15 DAE e $40 \mathrm{~g} \mathrm{ha}^{-1}$ aos 20 DAE; 4- $40 \mathrm{~g} \mathrm{ha}^{-1}$ de Mo aos 15 DAE e $40 \mathrm{~g} \mathrm{ha}^{-1}$ aos 25 DAE; 5- $40 \mathrm{~g} \mathrm{ha}^{-1} \mathrm{de}$ Mo aos 15 DAE e $40 \mathrm{~g} \mathrm{ha}^{-1}$ aos 30 DAE; 6- $80 \mathrm{~g} \mathrm{ha}^{-1}$ de Mo aos 20 DAE; 7- $40 \mathrm{~g} \mathrm{ha}^{-1}$ de Mo aos 20 DAE e $40 \mathrm{~g} \mathrm{ha}^{-1}$ aos 25 DAE; 8 - $40 \mathrm{~g} \mathrm{ha}^{-1}$ de Mo aos 20 DAE e $40 \mathrm{~g} \mathrm{ha}^{-1}$ aos 30 DAE; 9- $80 \mathrm{~g} \mathrm{ha}^{-1}$ de Mo aos 25 DAE; e 10- $40 \mathrm{~g} \mathrm{ha}^{-1}$ de Mo aos 25 DAE e $40 \mathrm{~g} \mathrm{ha}^{-1}$ aos 30 DAE.

Cada unidade experimental foi constituída de cinco linhas de $5 \mathrm{~m}$ de comprimento, espaçadas $0,5 \mathrm{~m}$ entre si. A parcela útil, com $6 \mathrm{~m}^{2}$, foi formada por três linhas centrais, excluindo-se, como bordadura, as linhas externas e meio metro de cada extremidade. Os dados de componentes da produção foram obtidos em duas linhas, com área total de $4 \mathrm{~m}^{2}$. A terceira linha foi usada para a coleta de dados do índice SPAD.

Os tratamentos receberam nos experimentos adubação uniforme com $300 \mathrm{~kg} \mathrm{ha}^{-1}$ da fórmula 08-28-16 no sulco de plantio. Não houve aplicação adicional de nitrogênio em cobertura. A fonte de Mo utilizada nos dois experimentos foi o molibdato de amônio.

O preparo do solo foi realizado de forma convencional, com uma aração e duas gradagens leves e posterior abertura de sulcos. Foram realizados dois cultivos para o controle de plantas daninhas, bem como os tratamentos fitossanitários necessários para manter os ensaios livres de pragas e de doenças durante todo o ciclo da cultura. Quando necessária, foi realizada irrigação por aspersão nos dois experimentos.

$\mathrm{Na}$ ocasião da colheita, determinaram-se, nos dois experimentos, o "stand" final, o número de vagens $\mathrm{m}^{-2}$, o número de grãos por vagem, a massa de $100 \mathrm{se}$ mentes e a produtividade de grãos $\mathrm{em} \mathrm{kg} \mathrm{ha}^{-1}$, com teor de umidade ajustado para $13 \%$.

$O$ índice SPAD foi determinado no estádio de florescimento pleno. As leituras foram efetuadas na primeira folha trifoliolada completamente desenvolvida, a partir do ápice da planta, em dez plantas, e em uma folha por planta, de uma das linhas centrais da área útil. As leituras foram realizadas no início da manhã com clorofilômetro modelo Minolta SPAD-502.

Os dados foram submetidos à análise de variância conjunta, de acordo com o critério da relação máxi- 
ma de 7:1 entre os quadrados médios dos resíduos dos experimentos (GOMES, 1985), sendo obtidos nove contrastes ortogonais, considerando o teste $\mathrm{F}$ a $5 \%$ de probabilidade:

1. Testemunha $v s$ Outros

2. Início 15 + Início 20 vs Início 25

3. Início 15 vs Início 20

4. Início 15 dose única vs Início 15 doses parceladas

5. Dose parcelada $15 / 20$ vs Dose parcelada (15/25 + 15/30)

6. Dose parcelada 15/25 vs Dose parcelada 15/30

7. Início 20 dose única $v s$ Início 20 dose parcelada

8. Dose parcelada 20/25 vs Dose parcelada 20/30

9. Início 25 dose única vs Dose parcelada 25/30

\section{RESULTADOS E DISCUSSÃO}

Pela análise conjunta dos experimentos I e II verificou-se efeito significativo $(\mathrm{P} \leq 0,05)$ da interação EXP x TRAT sobre o rendimento de grãos do feijoeiro. Esse comportamento, entretanto, não se repetiu para as demais características avaliadas (Tabela 1).

\section{"Stand" Final}

O contraste Testemunha vs Outros mostra que não houve efeito significativo da aplicação foliar de Mo sobre essa característica. Quanto às diferentes épocas de aplicação foliar desse nutriente, o contraste Dose parce- lada 15/25 vs Dose parcelada 15/30 mostrou-se signifi-cativo $(\mathrm{P} \leq 0,05)$ (Tabela 2$)$. Porém, em geral, todos os "stands" obtidos estão dentro da faixa considerada adequada para o feijão, isto é, entre 200 mil e 375 mil plantas ha ${ }^{-1}$ (VIEIRA, 1978).

\section{Produtividade de grãos}

No experimento de verão-outono (experimento I), observou-se efeito significativo $(\mathrm{P} \leq 0,01)$ da aplicação foliar de Mo sobre a produtividade do feijoeiro, que aumentou de 902 para $2.558 \mathrm{~kg} \mathrm{ha}^{-1}$, com incremento de $183,6 \%$ quando se comparou a testemunha (dose 0 ) com a média dos tratamentos que receberam $80 \mathrm{~g} \mathrm{ha}^{-1}$ de Mo (Testemunha vs Outros) (Tabela 3).

Da mesma forma, no experimento de invernoprimavera (experimento II), a aplicação do Mo aumentou significativamente o rendimento de grãos em aproximadamente $46 \%$, com a testemunha produzindo 1.833 $\mathrm{kg} \mathrm{ha}^{-1}$ e os tratamentos que receberam aplicação foliar de Mo, $2.680 \mathrm{~kg} \mathrm{ha}^{-1}$ (Testemunha vs Outros). Esse aumento de produção devido à adubação foliar de Mo pode ser resultado da influência desse micronutriente sobre as enzimas nitrogenase, responsável pela fixação biológica do $\mathrm{N}_{2}$, e redutase do nitrato, responsável pela redução do nitrato a nitrito, aumentando suas atividades, de modo a possibilitar maior aproveitamento do $\mathrm{N}$ e melhor qualidade nutricional das plantas, conforme relatado por Coelho et al. (1998), Vieira et al. (1998) e Zimmer e Mendel (1999).

TABELA 1 - Resumo da análise de variância conjunta dos dados de "stand" final (NUPLAN), número de vagem $\mathrm{m}^{-2}$ (VAGMT), número de sementes por vagem (SEMVAG), massa de 100 sementes (MACEM), produtividade (PROD) e índice SPAD (SPAD), do feijoeiro, nos experimentos I e II.

\begin{tabular}{lccccccc}
\hline \multirow{2}{*}{ FV } & GL & \multicolumn{7}{c}{ Quadrados Médios } \\
\cline { 3 - 7 } & & NUPLAN & VAGMT & SEMVAG & MACEM & PROD & SPAD \\
\hline BLO/EXP & 6 & $347005116^{\text {n.s. }}$ & $642,14^{\text {n.s. }}$ & $0,07^{\text {n.s. }}$ & $1,52^{\text {n.s. }}$ & $499547,16^{* *}$ & $17,99^{* *}$ \\
EXP & 1 & $6261376000^{\text {n.s. }}$ & $167490,8^{* *}$ & $11,12^{* *}$ & $159,32^{* *}$ & $821397,40^{*}$ & $452,20^{* *}$ \\
TRAT & 9 & $4356814000^{\text {n.s. }}$ & $8502,52^{* *}$ & $0,17^{\text {n.s. }}$ & $3,32^{* *}$ & $1362281^{* *}$ & $59,27^{* *}$ \\
EXPxTRAT & 9 & $1178203000^{\text {n.s. }}$ & $1368,96^{\text {n.s. }}$ & $0,02^{\text {n.s. }}$ & $1,79^{\text {n.s. }}$ & $267344,60^{*}$ & $7,44^{\text {n.s. }}$ \\
Resíduo & 54 & 3328688889 & 1766,70 & 0,08 & 0,97 & 105538,55 & 5,56 \\
\hline CV $(\%)$ & & 19,14 & 14,06 & 5,64 & 5,15 & 15,26 & 7,03 \\
\hline
\end{tabular}

n.s.: Não significativo ao nível de $5 \%$ de probabilidade

* $\mathrm{e}^{* *}$ : Significativo aos níveis de $5 \%$ e $1 \%$ de probabilidade, respectivamente

Ciênc. agrotec., Lavras, v. 28, n. 5, p. 1092-1098, set.out., 2004 
TABELA 2 - Contrastes estimados para "stand" final (NUPLAN), massa (g) de 100 sementes (MACEM), número de vagem $\mathrm{m}^{-2}$ (VAGMT), número de sementes por vagem (SEMVAG) e índice SPAD (SPAD) do feijoeiro, cv. Meia Noite, em função de doses e épocas de aplicação foliar de Mo.

\begin{tabular}{cccccc}
\hline \multirow{2}{*}{ Contrastes } & \multicolumn{5}{c}{ Diferenças } \\
\cline { 2 - 5 } & NUPLAN & MACEM & VAGMT & SEMVAG & SPAD \\
\hline 1 & $1,562^{\text {n.s. }}$ & $1,72^{* *}$ & $98^{* *}$ & $0,43^{* *}$ & $8,45^{* *}$ \\
2 & $15,469^{\text {n.s. }}$ & $0,03^{\text {n.s. }}$ & $20^{\text {n.s. }}$ & $0,01^{\text {n.s. }}$ & $0,58^{\text {n.s. }}$ \\
3 & $1,641^{\text {n.s. }}$ & $0,16^{\text {n.s. }}$ & $5^{\text {n.s. }}$ & $0,03^{\text {n.s. }}$ & $0,60^{\text {n.s. }}$ \\
4 & $28,646^{\text {n.s. }}$ & $0,85^{*}$ & $6^{\text {n.s. }}$ & $0,03^{\text {n.s. }}$ & $0,01^{\text {n.s. }}$ \\
5 & $5,000^{\text {n.s. }}$ & $0,06^{\text {n.s. }}$ & $2^{\text {n.s. }}$ & $0,03^{\text {n.s. }}$ & $0,97^{\text {n.s. }}$ \\
6 & $63,125^{*}$ & $0,63^{\text {n.s. }}$ & $16^{\text {n.s. }}$ & $0,18^{\text {n.s. }}$ & $0,99^{\text {n.s. }}$ \\
7 & $21,563^{\text {n.s. }}$ & $0,15^{\text {n.s. }}$ & $1^{\text {n.s. }}$ & $0,12^{\text {n.s. }}$ & $0,23^{\text {n.s. }}$ \\
8 & $56,250^{\text {n.s. }}$ & $0,33^{\text {n.s. }}$ & $9^{\text {n.s. }}$ & $0,06^{\text {n.s. }}$ & $0,35^{\text {n.s. }}$ \\
9 & $3,437^{\text {n.s. }}$ & $0,67^{\text {n.s. }}$ & $9^{\text {n.s. }}$ & $0,02^{\text {n.s. }}$ & $0,53^{\text {n.s. }}$ \\
\hline
\end{tabular}

n.s.: Não significativo ao nível de $5 \%$ de probabilidade; *e**: Significativo aos níveis de $5 \%$ e $1 \%$ de probabilidade, respectivamente

1-Testemunha vs Outros; 2- Início 15 + Início 20 vs Início 25; 3- Início 15 vs Início 20; 4- Início 15 dose única vs Início 15 doses parceladas; 5- Dose parcelada 15/20 vs Dose parcelada (15/25 + 15/30); 6- Dose parcelada 15/25 vs Dose parcelada 15/30; 7- Início 20 dose única $v s$ Início 20 dose parcelada; 8- Dose parcelada 20/25 vs Dose parcelada 20/30; 9- Início 25 dose única vs Dose parcelada 25/30.

TABELA 3 - Contrastes estimados e respectivas diferenças para a produtividade $\left(\mathrm{kg} \mathrm{ha}^{-1}\right)$ do feijoeiro, cv. Meia Noite, em função de doses e épocas de aplicação foliar de Mo, nos experimentos de verão-outono (I) e de invernoprimavera (II).

\begin{tabular}{ccccc}
\hline Contrastes & I & Diferença & II & Diferença \\
\hline 1 & $902 v s 2.558$ & $1.656^{* *}$ & $1.833 v s 2.680$ & $847^{* *}$ \\
2 & 2.641 vs 2269 & $372^{* *}$ & $2.688 v s 2.653$ & $35^{\text {n.s. }}$ \\
3 & $2.705 v s 2.556$ & $149^{\text {n.s. }}$ & $2.705 v s 2.665$ & $40^{\text {n.s. }}$ \\
4 & $2.572 v s 2.749$ & $177^{\text {n.s. }}$ & $2.564 v s 2.752$ & $188^{\text {n.s. }}$ \\
5 & $2.695 v s 2.776$ & $81^{\text {n.s. }}$ & $2.757 v s 2.749$ & $8^{\text {n.s. }}$ \\
6 & $2.768 v s 2.784$ & $16^{\text {n.s. }}$ & $2.592 v s 2.906$ & $314^{\text {n.s. }}$ \\
7 & $2.335 v s 2.666$ & $331^{\text {n.s. }}$ & $2.812 v s 2.592$ & $220^{\text {n.s. }}$ \\
8 & $2.788 v s 2.544$ & $244^{\text {n.s. }}$ & $2.531 v s 2.653$ & $122^{\text {n.s. }}$ \\
9 & $2.139 v s 2.400$ & $261^{\text {n.s. }}$ & $2.745 v s 2.561$ & $184^{\text {n.s. }}$ \\
\hline
\end{tabular}

n.s.: Não significativo ao nível de $5 \%$ de probabilidade; *e**: Significativo aos níveis de $5 \%$ e $1 \%$ de probabilidade, respectivamente

1-Testemunha vs Outros; 2- Início 15 + Início 20 vs Início 25; 3- Início 15 vs Início 20; 4- Início 15 dose única vs Início 15 doses parceladas; 5- Dose parcelada 15/20 vs Dose parcelada $(15 / 25+15 / 30)$; 6- Dose parcelada 15/25 vs Dose parcelada 15/30; 7- Início 20 dose única vs Início 20 dose parcelada; 8- Dose parcelada 20/25 vs Dose parcelada 20/30; 9- Início 25 dose única vs Dose parcelada 25/30. 
O rendimento de grãos do feijoeiro também foi influenciado $(\mathrm{P} \leq 0,01)$ pela época de aplicação foliar do Mo no experimento I. Essa influência foi detectada pelo contraste 2 (Início aos $15 \mathrm{DAE}$ + Início aos 20 DAE vs Início aos 25 DAE) (Tabela 3), em que a aplicação precoce do micronutriente aumentou em 16\% (de 2.269 para $2.641 \mathrm{~kg} \mathrm{ha}^{-1}$ ) a produtividade da cultura em relação à sua aplicação em época mais tardia. Com esses resultados pode-se inferir que a aplicação foliar do Mo é mais eficiente quando efetuada nos estádios mais iniciais do desenvolvimento, em comparação à sua aplicação tardia. Neves et al. (1982) citam que a atividade da nitrogenase e da redutase do nitrato no feijoeiro iniciase aproximadamente a partir do $14^{\circ}$ dia após o plantio (DAP), com aumentos progressivos até atingir o pico de atividade no $20^{\circ}$ DAP. A partir dessa fase do ciclo, ocorrem quedas nas atividades dessas enzimas, sendo determinado o menor índice de atividade para ambas aos 42 DAP. O suprimento dessa demanda mais elevada de Mo no início do ciclo provavelmente é a razão da obtenção de maiores produtividades pelos tratamentos que receberam Mo em aplicações mais precoces, nos quais essas enzimas se mantiveram ativas por maior período de tempo (PESSOA, 1998).

Outra vantagem da aplicação precoce do Mo via foliar é que a planta se encontra pouco desenvolvida, facilitando a aplicação e reduzindo os danos mecânicos sobre elas. Os tratamentos que receberam Mo apresentaram plantas mais verdes, mais vigorosas, com maior formação de órgãos florais. Apresentaram também plantas com senescência mais tardia das folhas, proporcionando maior período de enchimento dos grãos, podendo esse comportamento ser atribuído à maior atividade da enzima redutase do nitrato, tal como observado por Coelho et al. (1998) e por Vieira et al. (1998), bem como à maior fixação biológica do nitrogênio atmosférico. Por outro lado, as plantas do tratamento testemunha apresentaram-se pouco desenvolvidas, com folhas pequenas e cloróticas, menor número de flores e com senescência mais precoce no período de formação das sementes.

A época de aplicação e o parcelamento da dose de Mo avaliada $\left(80 \mathrm{~g} \mathrm{ha}^{-1}\right)$ não influenciaram significativamente a produtividade do feijão no experimento II (inverno-primavera) (Tabela 3). Tal fato pode ter ocorrido em razão de as épocas de aplicação do Mo terem sido fixadas em DAE e não em estádios de desenvolvimento, reduzindo a influência dos tratamentos sobre a cultura, quando comparados com o plantio de verãooutono, por causa do alongamento de seu ciclo proporcionado pela incidência de baixas temperaturas no plantio de inverno-primavera. Segundo Portes (1996), se baixas temperaturas ocorrerem imediatamente após a semeadura, podem impedir, reduzir ou atrasar a germinação das sementes e a emergência das plântulas, podendo resultar em baixas populações de plantas, e durante o crescimento vegetativo, baixas temperaturas podem reduzir a altura da planta e o crescimento dos ramos, conduzindo à produção de pequeno número de vagens por planta; conseqüentemente, haverá baixa produtividade.

Massa de 100 sementes, número de vagens $\mathrm{m}^{-2}$, número de sementes vagem $^{-1}$ e índice SPAD

A massa de 100 sementes, o número de vagens $\mathrm{m}^{-2}$, o número de sementes vagem ${ }^{-1}$ e o índice SPAD foram afetados positivamente pela adubação foliar com Mo $(P \leq 0,01)$. Essa observação pode ser confirmada com os resultados do contraste Testemunha vs Outros (Tabela 2), que apresentaram acréscimos de $9,5 \%, 62 \%, 8,5 \%$ e $28 \%$ na massa de 100 sementes, no número de vagens $\mathrm{m}^{-2}$, no número de sementes vagem $^{-1}$ e no índice SPAD, respectivamente, na média dos dois experimentos. A massa máxima de 100 sementes foi $18,13 \mathrm{~g}$, para o tratamento que não recebeu Mo, e 19,85 g para os tratamentos que receberam Mo. Resultados semelhantes foram obtidos por Amane (1997), que conseguiu aumento de $13 \%$ com a aplicação de Mo.

Pelo resultado do contraste Testemunha vs Outros observa-se que o número de vagens $\mathrm{m}^{-2}$ foi $\mathrm{o}$ componente de produção que apresentou melhor resposta à aplicação de Mo, aumentando de 158 , no tratamento que não recebeu Mo, para 256 vagens $\mathrm{m}^{-2}$, nos que receberam a dose de $80 \mathrm{~g} \mathrm{ha}^{-1}$ de Mo. O número de sementes por vagem aumentou de 4,84 no tratamento testemunha para 5,27 nos outros tratamentos adubados com Mo. Os incrementos obtidos nos componentes de produção em resposta ao Mo estão relacionados provavelmente ao seu efeito benéfico sobre a fixação do $\mathrm{N}_{2}$ e assimilação do $\mathrm{N}$-nítrico na planta, melhorando a nutrição nitrogenada. Por conseguinte, foram originadas plantas e folhas bem desenvolvidas, com coloração verde acentuada, maior número de órgãos florais, bem como retardamento da senescência de folhas, aumentando, assim, o período de enchimento dos grãos.

As diferentes épocas de aplicação foliar do Mo influenciaram significativamente $(\mathrm{P} \leq 0,05)$ o peso de 100 sementes (Tabela 2). O contraste Início 15 dose única vs Início 15 dose parcelada mostrou acréscimo de 
4,5\% na massa de 100 sementes, quando o Mo foi aplicado parcelado com início aos 15 DAE.

$\mathrm{O}$ número de vagens $\mathrm{m}^{-2}$ e de sementes vagem ${ }^{-1} \mathrm{e}$ o índice SPAD não apresentaram respostas significativas às diferentes épocas de aplicação foliar do Mo, em dose única ou parcelada.

Os tratamentos que não receberam aplicação de Mo apresentaram o valor máximo de 29,46 unidades SPAD, contra o valor máximo de 37,91 unidades para os tratamentos que receberam Mo. Araújo (2000), com base em leituras obtidas com o mesmo medidor SPAD, verificou que tanto a aplicação foliar de Mo aos 25 DAE quanto a adubação nitrogenada em cobertura proporcionaram aumento do teor de clorofila nas folhas. Contudo, esse aumento foi ainda mais elevado quando da combinação da aplicação de Mo com a de $\mathrm{N}$ em cobertura. $\mathrm{O}$ aumento do valor do índice SPAD devido à adubação foliar com Mo mostra a estreita relação entre o Mo e a intensidade de cor verde da planta, devido à maior absorção de $\mathrm{N}$ (VIEIRA et al., 1992), à maior síntese de clorofila (TAIZ e ZEIGER, 1998) e ao aumento da atividade fotossintética, possibilitando o aumento do rendimento (STONE et al., 2002).

\section{CONCLUSÕES}

A adubação foliar molíbdica, na dose de $80 \mathrm{~g} \mathrm{ha}^{-1}$, influencia positivamente os componentes da produção e a intensidade de cor verde das folhas do feijoeiro.

A aplicação foliar de Mo, com início aos 15 e 20 dias após a emergência, em dose única ou parcelada, aumenta a produtividade de grãos no cultivo de verãooutono. No cultivo de inverno-primavera, a produtividade aumenta com o Mo aplicado entre 15 e 30 dias após a emergência, em dose única ou parcelada.

\section{REFERÊNCIAS BIBLIOGRÁFICAS}

AMANE, M. I. V. Adubação nitrogenada e molíbdica da cultura do feijão (Phaseolus vulgaris L.) na Zona da Mata de Minas Gerais: efeitos de doses, calagem e rizóbio. 1997. 83 f. Tese (Doutorado em Fitotecnia) Universidade Federal de Viçosa, Viçosa, 1997.

AMANE, M. I. V.; VIEIRA, C.; NOVAIS, R. F.; ARAÚJO, G. A. de A. Adubação nitrogenada e molíbdica da cultura do feijão na Zona da Mata de Minas Gerais. Revista Brasileira de Ciência do Solo, Viçosa, v. 23, p. 643-650, 1999.

ARAÚJO, P. R. de A. Combinações de doses de nitrogênio e molibdênio na cultura do feijão
(Phaseolus vulgaris L.). 2000. 55 f. Tese (Doutorado em Fitotecnia) - Universidade Federal de Viçosa, Viçosa, 2000.

BERGER, P. G.; VIEIRA, C.; ARAÚJO, G. A. de A. Efeitos de doses e épocas de aplicação de molibdênio sobre a cultura do feijão. Pesquisa Agropecuária Brasileira, Brasília, v. 31, n. 7, p. 473-480, 1996.

COELHO, F. C.; VIEIRA, C.; MOSQUIM, P. R.; CASSINI, S. T. A. Nitrogênio e molibdênio nas culturas do milho e do feijão, em monocultivo e em consórcio: I efeitos sobre o feijão. Revista Ceres, Viçosa, v. 45, n. 260, p. 393-407, 1998.

GOMES, F. P. Curso de estatística experimental. 11. ed. Piracicaba: Nobel, 1985. 467 p.

NEVES, M. C. P.; FERNANDES, M. S.; SÁ, M. F. M. Assimilação de nitrogênio em plantas noduladas de Phaseolus vulgaris L. e Vigna unguiculata (L.) WALP. Pesquisa Agropecuária Brasileira, Brasília, v. 17, n. 5, p. 689-695, 1982

PESSOA, A. C. dos S. Atividades de nitrogenase e redutase do nitrato e produtividade do feijoeiro em resposta à adubação com molibdênio e fósforo. 1998. 151 f. Tese (Doutorado em Solos) - Universidade Federal de Viçosa, Viçosa, 1998.

PESSOA, A. C. S.; RIBEIRO, A. C.; CHAGAS, J. M.; CASSINI, S. T. A. Concentração foliar de molibdênio e exportação de nutrientes pelo feijoeiro "Ouro Negro" em resposta à adubação foliar com molibdênio. Revista Brasileira de Ciência do Solo, Viçosa, v. 24, p. 75-84, 2000.

PORTES, T. A. Ecofisiologia. In: ARAÚJO, R. S.; RAVA, C. A.; STONE, L. F.; ZIMMERMANN, M. J. O. Cultura do feijoeiro comum no Brasil. Piracicaba: POTAFÓS, 1996. p. 101-137.

STONE, L. F.; SILVA, G. de M. E.; MOREIRA, J. A. A. Uso de clorofilômetro SPAD-502 na estimativa do nitrogênio foliar específico e da produtividade do feijoeiro. In: CONGRESSO NACIONAL DE PESQUISA DE FEIJÃO, 7., 2002, Viçosa. Resumos... Viçosa: UFV, 2002. p. 743-746.

TAIZ, L.; ZEIGER, E. Plant physiology. 2. ed. [S.l.]: Sinauer Associates, 1998. 794 p. 
VIEIRA, C. Cultura do feijão. Viçosa: UFV, 1978. $146 \mathrm{p}$

VIEIRA, C.; NOGUEIRA, A. O.; ARAÚJO, G. A. de A. Adubação nitrogenada e molíbdica na cultura do feijão. Revista Agricultura, Piracicaba, v. 67, n. 2, p. 117-124, 1992.

VIEIRA, R. F.; CARDOSO, E. J. B. N.; VIEIRA, C.;

CASSINI, S. T. A. Foliar application of molybdenum in common beans: I. nitrogenase and reductase activities in a soil of high fertility. Journal Plant Nutrition, Monticello, v. 21, n. 1, p. 169-180, 1998.

ZIMMER, W.; MENDEL, R. Molybdenum metabolism in plants. Plant Biology, Stuttgard, v. 1, p. 160-168, 1999. 